\title{
Transient Structural Disorder as a Facilitator of Protein-Ligand Binding: Native H/D Exchange-Mass Spectrometry Study of Cellular Retinoic Acid Binding Protein I
}

\author{
Hui Xiao and Igor A. Kaltashov \\ Department of Chemistry, University of Massachusetts at Amherst, Amherst, Massachusetts, USA
}

Binding of all-trans Retinoic Acid (RA) to Cellular Retinoic Acid Binding Protein I (CRABP I) does not result in significant changes of the protein tertiary structure, even though the binding site is inaccessible in a static apo-protein conformation. One of the proposed scenarios for the protein-ligand binding process invokes the notion of a flexible portal region adjacent to the binding site, while another model suggests that the requisite dynamic events are induced by dimerization of the apo-protein in solution. In this work, RA binding to CRABP I is studied in dilute solutions (low micro-molar range), where no dimer and/or oligomer formation occurs. Modulation of backbone dynamics within various segments of the protein by its ligand is assessed using a combination of hydrogen exchange, electrospray ionization mass spectrometry, and collision-induced dissociation of protein ions in the gas phase. Consistent with the portal model of ligand entry, several protein segments (most of them containing residues making hydrophobic contacts to RA in the holo-form of the protein) are flexible in the absence of the ligand. At the same time, the two segments containing arginine residues forming a salt bridge with RA form the least flexible region in the apo-form of the protein. Although the presence of RA in solution reduces flexibility of all protein segments, the largest effect is observed within four strands that form one of the two $\beta$-sheets enveloping a cavity which houses the ligand-binding site. These results are consistent with a model in which ligand binding occurs through a partially unstructured state of the protein with unobstructed access to the ligand-binding site. This intermediate (whose core is formed by the two stable arginine-containing strands) corresponds to a relatively low-energy local minimum on the apo-protein energy surface and is frequently sampled under native conditions. (J Am Soc Mass Spectrom 2005, 16, 869-879) (c) 2005 American Society for Mass Spectrometry

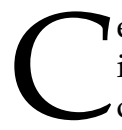
ellular retinoic acid binding protein I (CRABP I) is a member of a family of small soluble intracellular lipid binding proteins (iLBP) that bind hydrophobic ligands such as fatty acids, lipids, and retinoids [1]. CRABP I binds all-trans retinoic acid (RA), an active metabolite of vitamin $\mathrm{A}$, which controls a variety of cellular processes including differentiation, proliferation, and apoptosis in vertebrates from the embryonic stage throughout the entire adult life [2]. Despite being an important modulator of the pleiotropic effects of RA, CRABP I does not appear to interact directly with the nuclear retinoic acid receptor [3], and is likely to function as a sequestering agent rather than a transport protein [4]. This $15.5 \mathrm{kDa}$ protein contains 136 residues that form two five-stranded $\beta$-sheets (Figure 1). The first two strands are connected by a helixturn-helix motif, and all others by short reverse turns.

Published online April 13, 2005

Address reprint requests to Dr. I. A. Kaltashov, Department of Chemistry, University of Massachusetts, 710 North Pleasant Street, LGRT No. 701, Amherst, MA 01003, USA. E-mail: Kaltashov@chem.umass.edu
The two $\beta$-sheets are packed orthogonally to form a solvent-filled $\beta$-barrel. The ligand-binding pocket is located inside the barrel, with the carboxylate group of RA positioned innermost and the $\beta$-ionone ring lodged between the bottom half of $\alpha \mathbf{I I}$ and the reverse turns connecting $\beta 3$ to $\beta 4$ and $\beta 5$ to $\beta 6$. The RA-CRABP I interaction is predominantly hydrophobic, as the ligand forms ten contacts with nonpolar side chains, while there is only one group (carboxylate) involved in salt bridge-type interactions with the amino acid side chains of the protein [5]. The dissociation constant of the RA-CRABP I complex was estimated to be in the subnanomolar range based on the results of fluorescence measurements [3], while more recent measurements provided somewhat lower affinity estimates [6].

Comparisons of the apo- and holo-form structures of proteins in the iLBP family do not reveal significant openings large enough for the ligand entry and release $[7,8]$. Although the gap between $\beta 4$ and $\beta 5$ is apparently wider than the spacing between any other two $\beta$-strands, the access is obstructed by the side chains of amino acid residues forming these strands. This, as well 
(a)

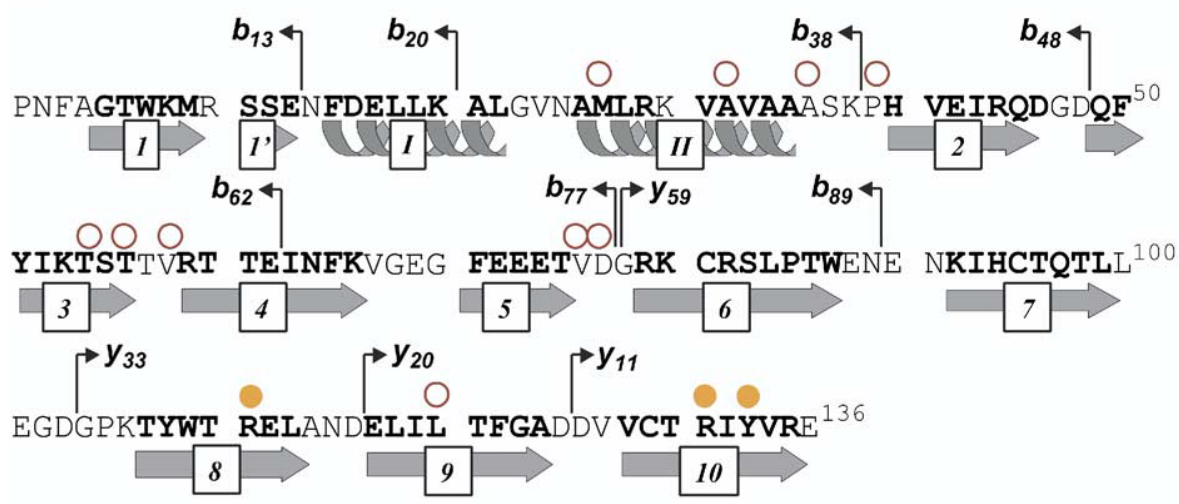

(b)

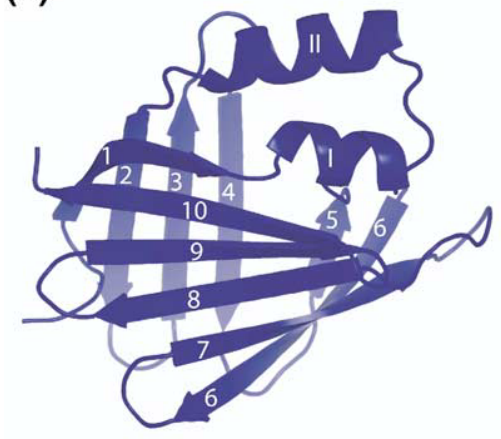

(c)

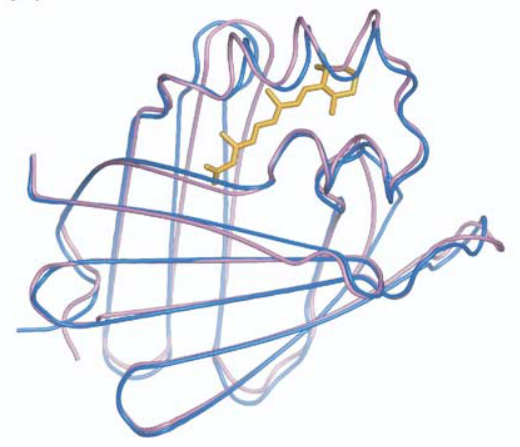

Figure 1. The amino acid sequence and a secondary structural map of apo-CRABP I ${ }^{5}$ (a). Residues forming a salt bridge to the carboxylate group of RA are indicated with filled circles. Open circles indicate the residues forming hydrophobic contacts with RA. Fragment ions discussed in the text are labeled with black arrows above the protein sequence. Tertiary structure of apo-CRABP I (b). Superimposed structures of the apo- (blue) and holo- (purple) forms of CRABP I (c).

as the lack of a significant conformational difference between the apo- and holo-forms of the protein suggests that certain dynamic events must play an important role in ligand binding to CRABP I. Jamison and coworkers noted increased susceptibility to proteolysis of several lipid-binding proteins in the absence of the ligand, a fact that was interpreted in terms of a more open conformation assumed by the apo-forms of the proteins in solution [9]. Thompson and coworkers suggested that the more open conformation of the apoprotein results from protein dimerization in solution, as suggested by the asymmetry of the dimeric unit in the crystal structure of apo-CRABP I [10]. However, the self-association model was challenged by recent NMR studies of CRABP II (a protein that shares $76 \%$ amino acid sequence identity with CRABP I), based on the absence of resonance signals assignable to the putative dimmer [11-13]. Gierasch and coworkers investigated CRABP I dimer formation in solution using relaxation dynamics measurements, confirming that CRABP I selfassociates at concentrations exceeding $1.2 \mathrm{mM}$ [14]. However, the RA-bound form of the protein formed dimers at this high concentration as well, while both apo- and holo-forms of the protein were suggested to exist predominantly in a monomeric form at sub-mM concentrations (based on the absence of dimer-specific resonances in the NMR spectra) [14].

An alternative to the self-association model of RA binding to CRABP I is a portal model, which invokes a notion of uneven distribution of conformational flexibility across the polypeptide chain. The existence of a flexible segment within the protein has been postulated. This segment serves as a transient opening to the binding site, thus allowing the ligand to enter the cavity with minimal changes in protein conformation $[15,16]$. Later revisions of the portal model by Hodson and Cistola led to the introduction of a dynamic portal model, in which the residues in the portal region are suggested to exhibit relatively large movements that enable ligand entry and release $[17,18]$. The portal region of CRABP $\mathrm{I}$ is thought to be comprised of a helix-turn-helix motif and two flanking $\beta$-hairpins (i.e., turns between $\beta 3 / \beta 4$ and $\beta 5 / \beta 6$ ). Reduced dynamics within this region in the holo-form of CRABP I effectively inhibits dissociation of RA from the protein in solution [14]. It is still unclear, however, if the increased backbone flexibility 
within the portal region is an intrinsic property of the protein or is induced (at least in part) by protein-protein interactions in solution. Indeed, all of the measurements discussed above were carried out at protein concentrations exceeding $0.1 \mathrm{mM}$, where the protein-protein interactions are expected to be quite significant, even though a monomeric form of CRABP I is predominant.

Protein dynamics and binding characteristics can often be probed at concentration levels close to or even below the endogenous levels, using a variety of strategies based on electrospray ionization mass spectrometry (ESI MS) [19]. Recently, we demonstrated that hydrogen/deuterium exchange (HDX) with ESI MS detection is a particularly effective tool to probe CRABP I backbone dynamics [20] and how it is affected by RA $[21,22]$. Modulation of protein backbone flexibility by ligand binding is reflected in measured HDX rates and the precision of such measurements allows RA to be distinguished from its isomers [6]. Identification of protein segments whose dynamics is most affected by ligand binding can be carried out in HDX MS experiments by measuring deuterium content of various fragment ions produced by collision-activated dissociation (CAD) of intact protein ions in the gas phase [21]. Such experiments provide a means to probe dynamics within distinct protein regions and determine ligand effect on their stability in relatively dilute solutions (sub- and low- $\mu \mathrm{M}$ range) under near-native or mildly denaturing conditions.

In this paper, we use HDX ESI MS and HDX ESI CAD MS to probe conformational stability of CRABP I and its segments under near-native conditions and at a concentration that is near its endogenous level. The differing levels of amide protection of the structural segments allow the conformational stability of this protein to be mapped and the possible sites of ligand entry to be identified. The conformational stability map of the apo-protein suggests that it frequently samples a partially unstructured state with unobstructed access to the ligand-binding site. A core of this intermediate state is formed by the two stable arginine-containing strands, which provide a binding template, or a scaffold, and also guide a RA anion into the binding site by creating an attractive electrostatic potential gradient, as proposed originally by Gierasch and coworkers [23].

\section{Experimental}

\section{Materials}

CRABP I samples were generously provided by Professor L. M. Gierasch (University of Massachusetts at Amherst). All-trans retinoic acid was purchased from Sigma-Aldrich Chemical Company (St. Louis, MO). Deuterium oxide (99.9 \% pure) was purchased from Cambridge Isotope Laboratories, Inc. (Andover, MA). All other chemicals and solvents used in this work were of analytical grade or higher.

\section{Mass Spectrometry}

ESI mass spectra of CRABP I at various protein concentrations were acquired with a JMS-700 MStation (JEOL, Tokyo, Japan) magnetic sector mass spectrometer equipped with a standard electrospray source. Samples were continuously infused at a $5 \mu \mathrm{l} / \mathrm{min}$ flow rate. All mass spectra were acquired under the mildest possible desolvating conditions in the ESI interface region (orifice/ring lens voltage $0 / 60 \mathrm{~V}$; desolvating plate/orifice temperature $80 / 60^{\circ} \mathrm{C}$ ) to minimize dissociation of noncovalently bound oligomeric species in the gas phase. Mass spectra were acquired by scanning the magnet at a $5 \mathrm{~s} /$ decade rate. Each recorded spectrum was an average of 50-100 scans. CRABP I concentrations were calculated by measuring absorption at $280 \mathrm{~nm}$ with a UV 1601PC (Shimadzu Corp., Tokyo, Japan) UV-Vis spectrophotometer; an extinction coefficient of 20,970 $\mathrm{M}^{-1} \mathrm{~cm}^{-1}$ was used to calculate the protein concentration $[24]$.

\section{Mass Spectrometry: HDX MS and HDX CAD MS Measurements}

All HDX MS measurements were carried out with an APEX-III Fourier transform ion cyclotron resonance mass spectrometer (Bruker Daltonics, Inc., Billerica, MA) equipped with a $4.7 \mathrm{~T}$ actively shielded magnet and a standard ESI source.

Collisional-activated dissociation of protein ions was achieved by increasing the capillary-exit potential from 90 to $300 \mathrm{~V}$ and increasing the hexapole accumulation time from 0.6 to $2 \mathrm{~s}$. Eight scans (with a total acquisition time of ca. $1 \mathrm{~min}$ ) were averaged to record each CAD spectrum to insure adequate signal-to-noise ratio. The CAD-generated fragment ions were initially identified based on their mass, and this assignment was further verified by mass-selecting the fragment ion of interest and inducing its dissociation in the ICR cell of the mass spectrometer to obtain amino acid sequence information.

All labile hydrogen atoms of CRABP I were replaced with deuterium in several cycles of protein incubation in ${ }^{2} \mathrm{H}_{2} \mathrm{O}$ at $\mathrm{pD} \sim 3$ (uncorrected for the isotope effect) and $45^{\circ} \mathrm{C}$ for $20 \mathrm{~min}$ followed by preconcentration of the protein solution at $4{ }^{\circ} \mathrm{C}$ using Centricon-10 (Millipore Corp., Beverly, MA) micro-concentrators. The complete deuteration of the protein was verified by ESI MS. The exchange reactions were initiated by a $1: 25$ $(\mathrm{vol} / \mathrm{vol})$ dilution of an aliquot of the protein stock solution in $10 \mathrm{mM} \mathrm{CH}_{3} \mathrm{COONH}_{4}(\mathrm{pH}$ 6.8) at room temperature to a final protein concentration of ca. $2 \mu \mathrm{M}$. HDX measurements of holo-CRABP I were carried out by adding RA to the exchange buffer at a 5-fold molar excess over the protein.

Average deuterium content of each fragment ion was calculated by using the centroid of its isotopic cluster and the protection level of each protein segment was calculated as a difference in ${ }^{2} \mathrm{H}$ content of the fragment 
ions that result from the dissociation of the peptide bonds flanking that segment. For example, protection of a segment spanning residues $i$ to $j$ will be calculated using ${ }^{2} \mathrm{H}$ contents of fragment ions $b_{i-1}$ and $b_{j}$ as:

$$
P_{i \rightarrow j}(\%)=\frac{N\left(b_{j}\right)-N\left(b_{i-1}\right)}{j-i} \times 100 \% .
$$

If the segment spanning residues $i$ to $j$ contained a proline residue, the denominator in the equation above was reduced by one to $j-i-1$.

To insure the absence of intermolecular hydrogen exchange in the gas phase (between the protein ions and the solvent molecules) throughout the extended

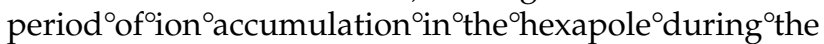
course of HDX CAD MS experiments, the deuterium content of surviving intact protein ions was compared with that of protein ions not subjected to collisional activation and prolonged trapping. The deviation is very $^{\circ}$ insignificant ${ }^{\circ}\left(\right.$ Figure $\left.^{\circ} 3 \mathrm{~b}\right)$, indicating ${ }^{\circ}$ that ${ }^{\circ}{ }^{\circ} o^{\circ}$ significant $^{\circ}$ post-exchang ${ }^{\circ}$ is ${ }^{\circ}$ occurring $^{\circ}$ in $^{\circ}$ the $^{\circ}$ ESI $^{\circ}$ interface region.

\section{Results}

\section{CRABP I Association in Solution}

Since the $\beta$-sheet content of CRABP I is very high, the protein is likely to be prone to association and aggregation in solution, particularly at elevated concentrations. Since dimer formation had been previously suggested to play a role in the protein conformational changes required for ligand binding, we explored the effect of protein concentration on the extent of its dimerization and (more generally) oligomerization. Endogenous concentration of CRABP I in tissues expressing this protein (e.g., limb buds, neuronal tissues, etc.) is estimated to be in the range $1-10 \mu \mathrm{M}$, a level that corresponds $9{ }^{\circ} \mathrm{ca} .{ }^{\circ} 10^{7}-10^{8^{\circ}}$ protein ${ }^{9}$ molecules ${ }^{9}$ per $^{\circ}$ cell [4]. The ${ }^{\circ} \mathrm{ESI}^{\circ} \mathrm{mass}^{\circ}$ spectrum ${ }^{\circ} \mathrm{of}^{\circ} \mathrm{a}^{\circ} 2^{\circ} \mu \mathrm{M}^{\circ}$ aqueous ${ }^{\circ}$ solution ${ }^{\circ}$ of apo-CRABP I contains only ion peaks corresponding to the ${ }^{\circ}$ monomeric $^{\circ}$ state $^{\circ}$ of ${ }^{\circ}$ the ${ }^{\circ}$ protein $^{\circ}$ (Figure $\left.{ }^{\circ} 2 a\right) .{ }^{\circ}$ The charge state distribution is narrow, and the average number of charges accommodated by the protein ions is low, indicating that the protein is compactly folded in solution. ${ }^{\circ} \mathrm{A}^{\circ}$ noticeable ${ }^{\circ}$ change ${ }^{\circ}$ in $^{\circ}$ the ${ }^{\circ}$ spectrum ${ }^{\circ}$ occurs when the protein concentration is increased ten-fold to $20^{\circ} \mu \mathrm{M}^{\circ}$ (Figure $\left.{ }^{\circ} 2 \mathrm{~b}\right) .{ }^{\circ} \mathrm{A}^{\circ}$ new $^{\circ}$ set $^{\circ}$ of $^{\circ}$ ion $^{\circ}$ peaks ${ }^{\circ}$ appears ${ }^{\circ}$ in $\mathrm{m} / \mathrm{z}$ range $2300-2800$. The mass of this ionic species exceeds ${ }^{\circ}$ that ${ }^{\circ}$ of $^{\circ} \mathrm{CRABP}^{\circ} \mathrm{I}^{\circ}$ by $^{\circ} \mathrm{a}^{\circ}{ }^{\circ}$ actor ${ }^{\circ}$ of ${ }^{\circ}$ two, ${ }^{\circ}$ and ${ }^{\circ}$ the average charge is +12 , suggesting that it corresponds to a protein dimer. Further increase of the protein concentration ${ }^{\circ}$ (to ${ }^{\circ} 50^{\circ} \mu \mathrm{M},{ }^{\circ}$ Figure $2 \mathrm{c}$ ) ${ }^{\circ}$ leads ${ }^{\circ}$ to ${ }^{\circ}$ broadening ${ }^{\circ}$ of ${ }^{\circ}$ the dimer ion peaks, as well as to the appearance of two new ionic species whose masses correspond to a CRABP I trimer $(\mathrm{m} / \mathrm{z} 2800-3300)$ and tetramer $(\mathrm{m} / \mathrm{z}$ $3300-3800)$. Finally, the ESI mass spectrum of a $100 \mu \mathrm{M}$ $\mathrm{CRABP}^{\circ}{ }^{\circ}$ solution ${ }^{\circ}$ (Figure $\left.2 \mathrm{~d}\right)^{\circ}$ Contains $^{\circ}$ ionic ${ }^{\circ}$ signal ${ }^{\circ}$ from penta- and hexameric protein species $(\mathrm{m} / \mathrm{z} 3800-4700)$.

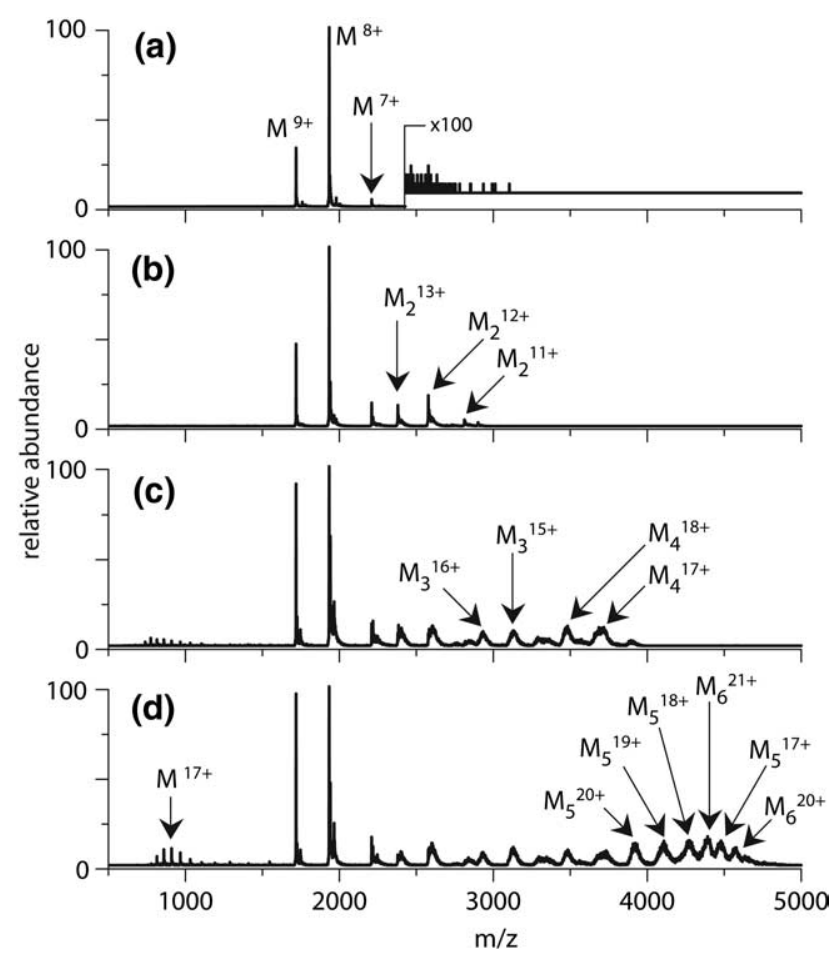

Figure 2. ESI mass spectra of apo-CRABP I in $10 \mathrm{mM}$ $\mathrm{CH}_{3} \mathrm{COONH}_{4}$ at a concentration of $2 \mu \mathrm{M}(\mathbf{a}), 20 \mu \mathrm{M}(\mathbf{b}), 50 \mu \mathrm{M}$ (c), and $100 \mu \mathrm{M}$ (d).

The oligomer ion peaks were notably absent from the spectra of CRABP I acquired under strongly denaturing conditions (50\% methanol by volume, acidified with $3 \%$ acetic acid, see Supplementary Material), suggesting that the appearance of multiple broad peaks in the high $\mathrm{m} / \mathrm{z}$ range (above 2500) under near-native conditions does indeed signal protein oligomerization in solution, rather than a gas-phase artifact.

Another peculiar feature of the spectra acquired under near-native conditions using elevated protein concentration is the appearance of monomeric protein ions with a high number of charges (a distribution centered around $m / z$ 900). Williams and coworkers have recently linked the appearance of such high charge density protein ions in ESI mass spectra acquired under native conditions to the asymmetric dissociation of noncovalently bound higher oligomers in the gas phase [25]. ${ }^{\circ}{ }^{\circ}{ }^{\circ}{ }^{\circ}$ example, ${ }^{\circ} a^{\circ}$ monomeric ${ }^{\circ}$ ion $^{\circ}$ carrying $^{\circ}$ seventeen charges can be a product of an asymmetric gas phase dissociation of a weakly bound hexamer carrying twenty-one charges (the complementary fragment ion, a pentamer carrying the four remaining charges will not be detected, as its $\mathrm{m} / \mathrm{z}$ falls well above 5000). These measurements indicate that even though the monomeric state of CRABP I remains predominant in the concentration range 10-100 $\mu \mathrm{M}$, self-association of the protein in solution becomes prominent under these conditions. Importantly, no self-association is observed at low- $\mu \mathrm{M}$ concentrations, suggesting that the conformational characteristics of CRABP I measured under 

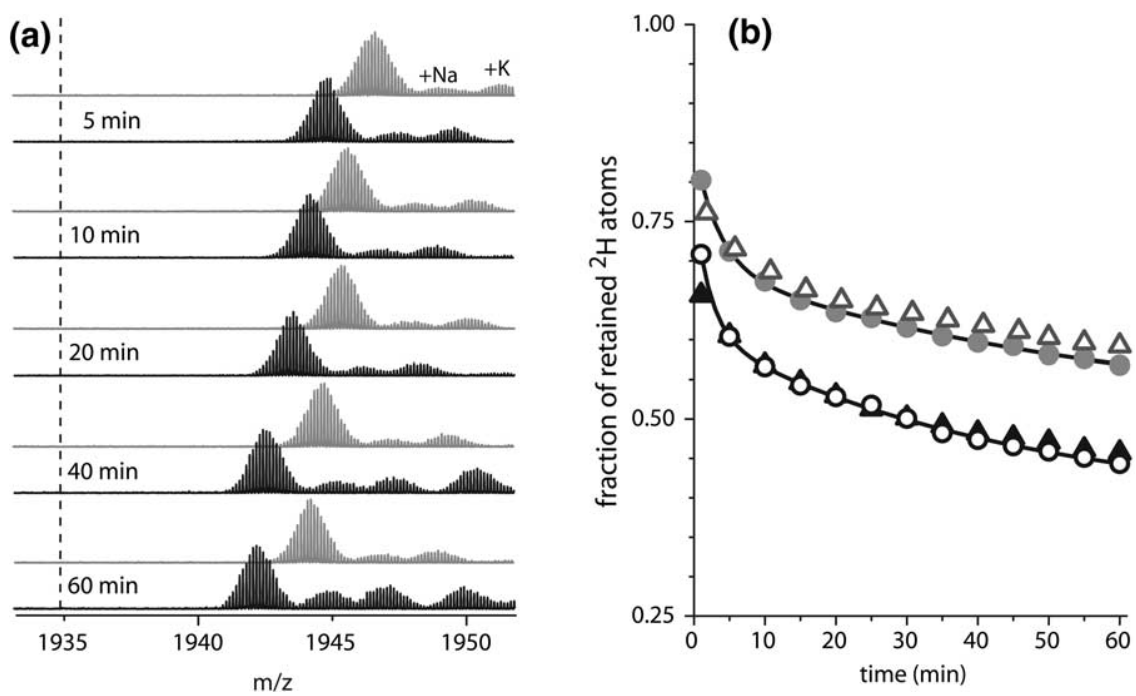

Figure 3. Evolution of isotopic clusters of intact (charge state +8 ) ions of CRABP I undergoing HDX in the absence (black traces) and presence (gray traces) of RA in the exchange buffer at $\mathrm{pH} 6.8$ (a). Dotted line indicates position of a centroid of an isotopic cluster of a fully exchanged protein (whose total ${ }^{2} \mathrm{H}$ content is the same as the solvent, i.e., $4 \%$ ). Plots of HDX kinetics based on the ${ }^{2} \mathrm{H}$ content of intact protein ions in HDX MS measurements (circles) and surviving molecular ions in HDX CAD MS experiments (triangles) in the absence (black symbols) and presence (gray) of RA in the exchange buffer $(\mathbf{b})$

these conditions will represent the intrinsic properties of the protein and will not be affected by its dimerization.

\section{HDX ESI MS of CRABP I: Ligand Binding and Backbone Flexibility}

HDX measurements of CRABP I were performed at room temperature at a protein concentration that excludes ${ }^{\circ}$ the ${ }^{\circ}$ ossibility ${ }^{\circ}$ f $^{\circ}$ dimer $^{\circ}$ formation ${ }^{\circ}$ (Figure 3$)^{\circ}$ and clearly indicate contributions from various dynamic processes. A relatively fast phase of the exchange $(\tau \leq$ $10 \mathrm{~min}$ ) affecting a limited number of amide hydrogen atoms ${ }^{\circ}$ corresponds $^{\circ}$ to $^{\circ}{ }^{\circ}$ ccal $^{\circ}$ structural $^{\circ}$ fluctuations ${ }^{\circ}[26]$, while the slower phase corresponds to partial unfolding of the protein (e.g., transitions to partially unstructured equilibrium states). The slowest phase of exchange, which is usually attributed to the very rare global unfolding events, is not observed on the time-scale of our experiment. Since this work aims at examining the role played by partially unstructured state(s) of CRABP I in ligand binding, we focus our attention on the intermediate phase of HDX (20-60 min). The difference in amide protection between the apo- and holo-forms of CRABP I remains nearly constant throughout this phase of $^{\circ}$ the ${ }^{\circ}$ exchange ${ }^{\circ}$ (Figure $3 \mathrm{~b}$ ), ${ }^{\circ}$ changing ${ }^{\circ}$ from $15^{\circ} \pm 3^{\circ}$ after $30 \mathrm{~min}$ of exchange to $16 \pm 3$ after $60 \mathrm{~min}$ (the total number of backbone amide hydrogen atoms in CRABP I is 131). It is important to note that the significant change in CRABP I backbone protection induced by RA is solely attributable to the changes of protein flexibility, as the protein-ligand binding does not include forma- tion of any hydrogen bonds involving backbone amide hydrogen atoms. A significant decrease of CRABP I flexibility in the presence of RA during the intermediate phase of exchange clearly indicates that sampling of a putative intermediate state(s) becomes much less frequent when the ligand is bound to the protein.

\section{HDX CAD MS of apo-CRABP I: Conformational Stability Map}

Measurements of flexibility distribution along the polypeptide chain were carried out by inducing fragmentation of CRABP I ions prior to MS detection (Figure $\left.{ }^{\circ} 4\right)^{\circ}{ }^{\circ} \mathrm{CAD}^{\circ}$ in $^{\circ}$ the ${ }^{\circ} \mathrm{ESI}^{\circ}$ interface ${ }^{\circ}$ region ${ }^{\circ}$ was $^{\circ}$ used $^{\circ}$ in this work to induce protein ion dissociation, as it eliminates the possibility of hydrogen scrambling within the activated protein ions prior to their dissociation $^{\circ}\left[21,{ }^{\circ} 27\right] .{ }^{\circ}$ Overall, ${ }^{\circ}$ nineteen ${ }^{\circ}$ fragment $^{\circ}$ ions ${ }^{\circ}$ have been consistently observed in all CAD mass spectra. Assignment of the observed fragment ions in the CAD spectrum was a relatively simple task because of the superior resolving power of a Fourier transform ion cyclotron resonance mass spectrometer (FT ICR MS). A clear distinction could be made between different fragment ions even if their isotopic clusters overlapped (e.g., $\left[y_{11}-\mathrm{H}_{2} \mathrm{O}\right]^{+}$and $y_{35}^{3+}$ in $^{\circ}$ Figure $^{\circ} 4$ ), ${ }^{\circ}$ based ${ }^{\circ}$ on ${ }^{\circ}$ the difference in their charge states.

Although in some instances CAD produces closely spaced clusters of fragment ions by inducing cleavages of neighboring peptide bonds (e.g., $b_{17}^{2+}$ and $b_{18}^{2+}$ ), no effort has been made in this work to determine protection of individual backbone amide groups. Deinzer and 


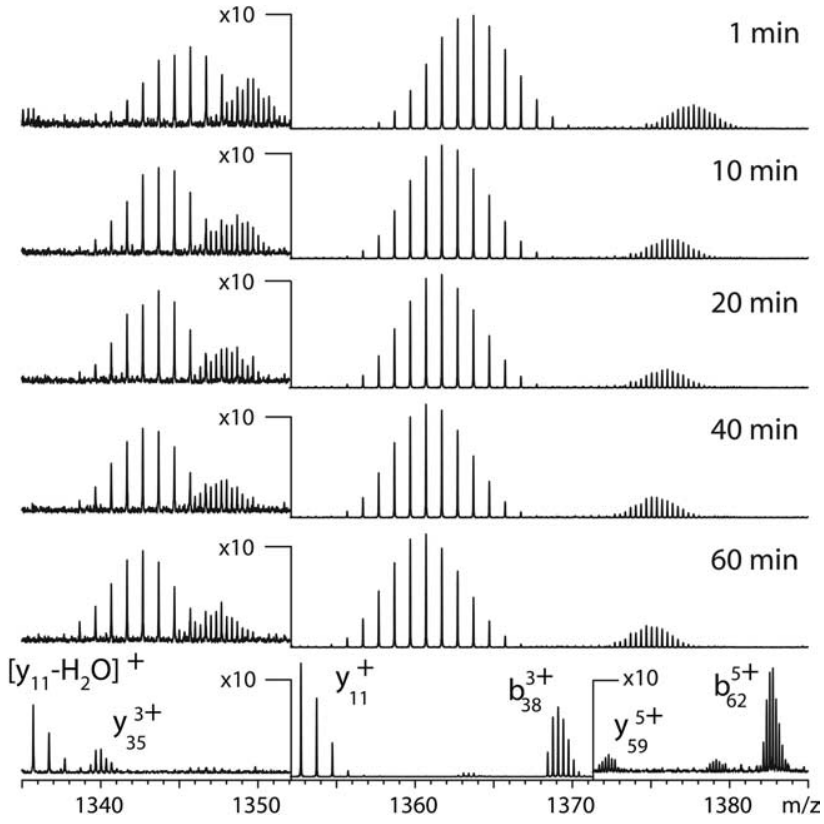

Figure 4. Evolution of isotopic clusters of several CAD-generated fragment ions of apo-CRABP I undergoing hydrogen exchange in solution. The bottom trace represents the isotopic distributions of unlabeled (essentially ${ }^{2} \mathrm{H}$-free) fragment ions of CRABP I.

coworkers have demonstrated that such measurements are not always reliable, since a peptide bond cleavage often involves local proton transfer from a chargebearing ${ }^{\circ}$ side $^{\circ}$ chain $^{\circ}$ to ${ }^{\circ} \mathrm{a}^{\circ}$ backbone $^{\circ}$ amide $^{\circ}$ group $^{\circ}[28]$. However, these charge-directed rearrangement reactions leading to a peptide bond dissociation are local and do not exert significant influence over the deuterium content of segments spanning several amino acid residues $^{\circ}[27]^{\circ}{ }^{\circ} \mathrm{To}^{\circ}$ construct $^{\circ}$ the ${ }^{\circ}$ protein $^{\circ}$ stability $^{\circ}$ map, eleven fragment ions that span the entire sequence of CRABP I have been chosen, providing a fairly uniform division of the polypeptide chain into structural segments ${ }^{\circ}$ (Figure $\left.1 a\right) .{ }^{\circ}$ Calculation ${ }^{\circ}{ }^{\circ}$ of the ${ }^{\circ}$ deuterium ${ }^{\circ}$ content of each structural segment was carried out by using the measured numbers of deuterium atoms retained by the fragment ions that "bracket" the segment, (e.g., $b_{77}$ and $b_{89}$ for the strand $\beta 6$ (see the Material and Methods section for more detail). For some protein segments ${ }^{2} \mathrm{H}$ content can be calculated in two different ways. For example, the deuterium content of $\beta 6$ can also be calculated as a difference between the sum of ${ }^{2} \mathrm{H}$ atoms retained by $y_{59}$ and $b_{89}$ fragments and the intact protein ion. ${ }^{\circ} \mathrm{As}^{\circ}$ shown $^{\circ}$ in $^{\circ}$ Figure $^{\circ}{ }^{\circ},{ }^{\circ}$ the ${ }^{\circ}$ two $^{\circ}$ different $^{\circ}$ ways $^{\circ}$ of calculating $\beta 6{ }^{2} \mathrm{H}$ content provide identical results, clearly ${ }^{\circ}$ indicating ${ }^{\circ}$ that ${ }^{\circ}$ the $^{\circ}$ calculated ${ }^{\circ}$ protection ${ }^{\circ}$ levels are independent of selection of bracketing fragment ions.

Deuterium content of each of the eleven protein segments has been calculated and plotted as a function of $^{\circ}$ exchange $^{\circ}$ time $^{\circ}$ in $^{\circ}$ solution $^{\circ}\left(\right.$ Figure $^{\circ} 6 \mathrm{a}$ ). ${ }^{\circ}$ The $^{\circ}$ plot clearly indicates that flexibility is distributed unevenly along the polypeptide chain. Two strands ( $\beta 8$ and $\beta 10)$ exhibit the highest levels of protection, which greatly exceed the average protection level of the intact protein. The most flexible regions of CRABP I in the absence of the ligand are helix $\alpha \mathbf{I I}$, and strands $\beta 4 / 5$ and $\beta 9$. Other segments of the protein have been determined to have intermediate levels of amide protection between these two extremes (see the color-coded stability map at the bottom $^{\circ}$ of $^{\circ}$ Figure $\left.^{\circ} 6 \mathrm{a}\right) .{ }^{\circ}$ Importantly, ${ }^{\circ}$ the ${ }^{\circ}$ two ${ }^{\circ}$ segments with the highest amide protection ( $\beta 8$ and $\beta 10)$ contain both amino acid residues whose side chains form a salt bridge to the carboxylate group of RA in the crystal structure of the holo-protein ( $\mathrm{Arg}^{111}$ in $\beta 8$ and $\mathrm{Arg}^{131}$ in $\beta 10)$, but none of the residues forming hydrophobic contacts $^{\circ}$ with $^{\circ}$ the $^{\circ}$ ligand $^{\circ}\left(\right.$ Figure $\left.^{\circ} 1\right)$.

\section{Conformational Stability Map of CRABP I in the Presence of $R A$}

The conformational stability map of the holo-form of CRABP I was obtained in the same way as discussed above for the apo-form using a five-fold molar excess of RA in the exchange buffer. The presence of RA in solution affected all eleven protein segments, although the extent of RA-induced protection increase varies significantly ${ }^{\circ}$ among ${ }^{\circ}$ the ${ }^{\circ}$ segments $^{\circ}$ (Figure $\left.{ }^{\circ} 6 \mathrm{~b}\right)$. The $^{\circ}$ most insignificant gain is observed within the strand $\beta 8$, while the biggest "winners" are segments $\beta 3 / 4, \beta 4 / 5, \beta 6$, and $\beta 7$. Four out of five strands represented by these segments form one of the two $\beta$-sheets enveloping the

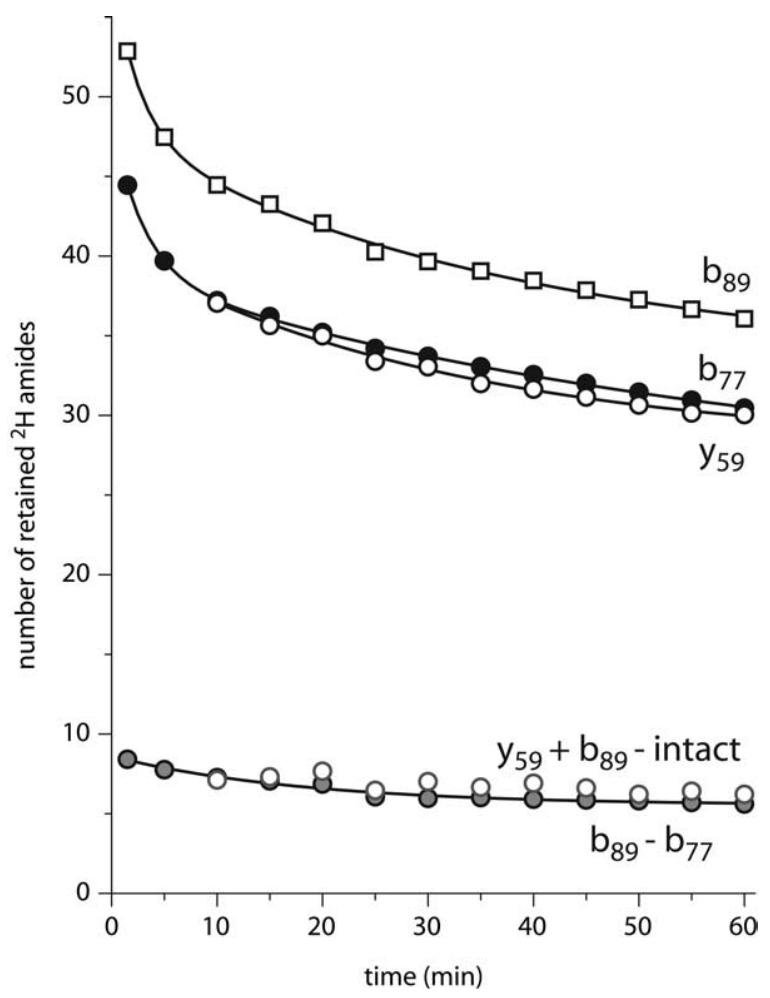

Figure 5. Time evolution of ${ }^{2} \mathrm{H}$ content of several fragment ions (top curves) used to calculate protection within the protein segment $\beta 6$ in two different ways (bottom curves). 

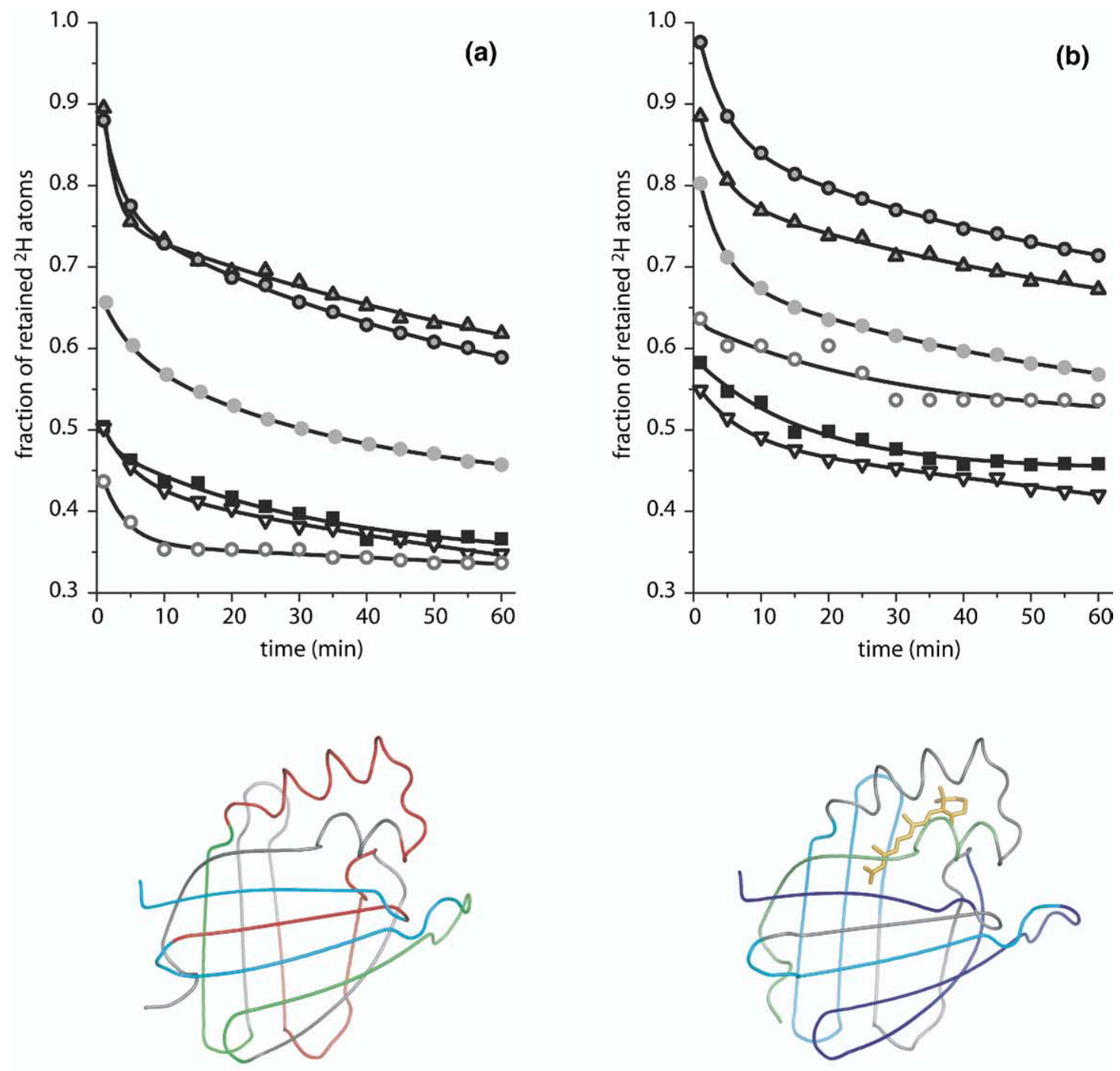

protection levels after 30 min of exchange reaction

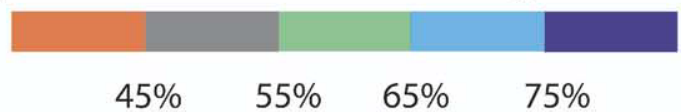

Figure 6. Protection of various segments of CRABP I in the absence (a) and presence (b) of RA in the exchange buffer: $\beta 4 / 5$ (open gray circles), $\beta 9$ (inverse triangles), $\alpha$ II (filled squares), $\beta 10$ (open black circles), $\beta \mathbf{8}$ (triangles), and intact protein (shaded gray circles). Color-coded stability maps of apo- and holo-CRABP I are shown at the bottom of each diagram.

solvent-filled cavity of the protein. The average increase of the amide protection level induced by RA is $20 \%$

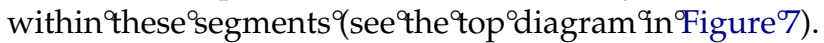
Among ${ }^{\circ}$ these ${ }^{\circ}$ four ${ }^{\circ}$ segments, ${ }^{\circ} \beta 4 / 5$ was ${ }^{\circ}$ the ${ }^{\circ}$ most ${ }^{\circ}$ flexible structural ${ }^{\circ}$ element ${ }^{\circ}$ in ${ }^{\circ}$ the ${ }^{\circ}$ apo-form ${ }^{\circ}$ of ${ }^{\circ} \mathrm{CRABP}^{\circ} \mathrm{I}^{\circ}$ (Figure $7,{ }^{\circ}$ bottom ${ }^{\circ}$ diagram).$^{\circ}$ Interestingly, ${ }^{\circ}$ the ${ }^{\circ} \beta$-turns ${ }^{\circ} \mathbf{3} / \mathbf{4}$ and $5 / 6$ are positioned strategically near the proposed portal region of the protein and are responsible for one half of all hydrophobic contacts between the protein and the ligand.

\section{Discussion}

The most contentious point in the two existing models of RA binding to CRABP I relates to the role played by protein dimerization in the ligand binding process. While the protein crystal structure by definition is biased towards the oligomerized state (the one producing the most efficient packing), and NMR measurements clearly suggest that a monomeric state of CRABP $\mathrm{I}$ is predominant in the sub-mM concentration range 

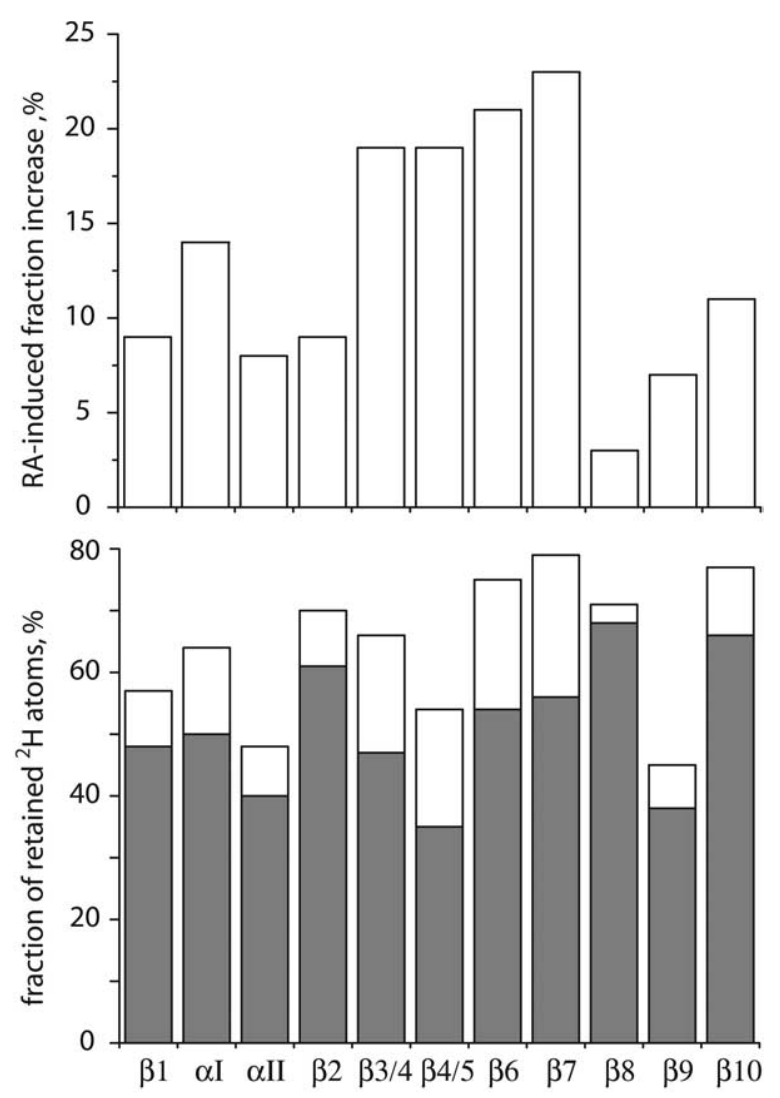

CRABP I structural elements

Figure 7. A bar graph representing fractions of amide ${ }^{2} \mathrm{H}$ retained by various segments of CRABP I following $30 \mathrm{~min}$ of HDX in the absence (gray bars) and presence (full-length bars) of RA in the exchange buffer (bottom diagram). Top diagram represents increase of amide protection for each segment induced by RA.

[14], ${ }^{\circ} a^{\circ}$ possibility $^{\circ}$ that $^{\circ} \mathrm{a}^{\circ}$ minor $^{\circ}$ fraction ${ }^{\circ}$ of ${ }^{\circ}$ the ${ }^{\circ}$ protein molecules do form dimers even in relatively dilute solutions cannot be discounted. It could be possible in this case that protein dimerization, however ineffective, catalyzes and directs RA binding to CRABP I by inducing the requisite conformational changes. Our concentration-dependent study of CRABP I self-association clearly indicates that a significant proportion of the protein does exist in various oligomeric states (including a dimeric one) at concentrations as low as tens of $\mu \mathrm{M}^{\circ}$ (Figure $2 \mathrm{~b}-\mathrm{d}$ ) ${ }^{\circ}$ However, ${ }^{\circ}{ }^{\circ}{ }^{\circ}$ self-association ${ }^{\circ}$ occurs in the ${ }^{\circ}$ low $-\mu \mathrm{M}^{\circ}$ range $^{\circ}$ (Figure $2 \mathrm{a}$ ), ${ }^{\circ}$ apparently ${ }^{\circ}$ having ${ }^{\circ}$ no effect on the ligand-binding properties of CRABP I. It follows then that any dynamic events within the protein that facilitate ligand binding under these conditions are attributable solely to the intrinsic properties of CRABP I and cannot possibly be induced by dimer formation.

\section{Dynamics of apo-CRABP I and the Ligand- Binding Sites of the Protein}

The ligand-binding region is buried within CRABP I in a mostly hydrophobic environment. Specific residues involved in the interaction between RA and CRABP I are not distributed evenly throughout the protein, with 9 out of 10 residues making hydrophobic contacts to the ligand being located at or near the postulated portal region of ${ }^{\circ}$ the ${ }^{\circ}$ protein $^{\circ}\left(\right.$ Figure $\left.^{\circ} 1\right) .^{\circ}$ The $^{\circ}$ corresponding structural segments $(\alpha \mathbf{I I}$ and $\beta 4 / 5)$ are among the three segments exhibiting highest rates of HDX in the absence of ${ }^{\circ} \mathrm{RA}^{\circ}$ (Figures $\left.{ }^{\circ} 6 \mathrm{a}^{\circ}{ }^{\circ}{ }^{\circ}{ }^{\circ} 7\right) .{ }^{\circ}$ This $^{\circ}$ observation ${ }^{\circ}$ is $^{\circ}$ in ${ }^{\circ}$ agreement with the earlier NMR measurements by Gierasch and coworkers, who identified these segments as the most flexible in apo-CRABP I at elevated concentrations and suggested that their flexibility must be critical for

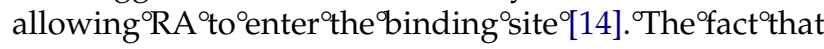
a significant proportion of the residues making hydrophobic contacts to RA are located in these segments clearly indicates that native hydrophobic interactions do not play a decisive role during the initial stages of the protein-ligand binding.

The increased backbone flexibility at and around the portal region contrasts sharply with low conformational mobility of the two segments containing amino acid residues whose positively charged side chains interact with a carboxylate group of the ligand by forming a salt bridge-like structure ( $\operatorname{Arg}^{111}$ in $\beta 8$ and $\operatorname{Arg}^{131}$ with some involvement of $\mathrm{Tyr}^{133}$ in $\beta 10$ ). Backbone amide protection within these segments is remarkably high even in the absence of RA, making them the two least mobile segments ${ }^{\circ}$ of apo-CRABP ${ }^{\circ}$ (Figure $\%$ a). It $^{\circ}$ was ${ }^{\circ}$ previously proposed that the RA anion ${ }^{\circ}$ entry into the protein ${ }^{\circ}$ cavity may be guided by an attractive electrostatic potential created by several positively charged residues extending ${ }^{\circ}$ from ${ }^{\circ}$ the ${ }^{\circ}$ cavity ${ }^{\circ}$ to ${ }^{\circ}$ the ${ }^{\circ}$ entry ${ }^{\circ}$ site, ${ }^{\circ}$ which ${ }^{\circ}$ include both $\operatorname{Arg}^{111^{\circ}}$ and ${ }^{\circ} \operatorname{Arg}^{131^{\circ}}$ [23]. ${ }^{\circ}$ The $^{\circ}$ notion ${ }^{\circ}$ of ${ }^{\circ}$ ligand-guiding potential was supported in a recent study of an apoCRABP II mutant, in which one of the charged residues was ${ }^{\circ}$ replaced ${ }^{\circ}$ with $^{\circ} a^{\circ}$ neutral ${ }^{\circ}$ one $[13]$.

The remarkable conformational stability of $\beta 8$ and $\beta 10$, as well as their role in creating an attractive electrostatic potential, may indicate that these two segments retain their native-like structure in the partially unstructured state of the protein with unobstructed access to the ligand-binding site and a stable template for initial protein-ligand binding. We also note that the strand $\beta 7$, flanking $\beta 8$ and its own neighbor, $\beta 6$, are relatively inflexible in the apo-state of the protein as well, even though they are remote from the ligand-

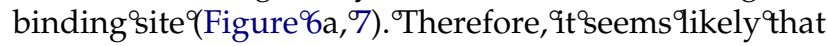
$\beta 6$ and $\beta 7$, together with $\beta 8$ and $\beta 10$, are also a part of the stable core of the activated state of CRABP I that is critical for ligand binding.

One very surprising result of our studies is a very high degree of conformational mobility of $\beta 9$, a strand that is, in the native state of the protein, positioned between the two strands forming a stable ligand-binding $^{\circ}$ template, ${ }^{\circ} \beta 8$ and $^{\circ} \beta 10$ (Figure ${ }^{\circ} 6 a$ ). ${ }^{\circ}$ The $^{\circ}$ increased flexibility of this strand has also been noted in the earlier ${ }^{\circ} \mathrm{NMR}^{\circ}$ measurements ${ }^{\circ}[14] . .^{\circ}$ One ${ }^{\circ}$ possible ${ }^{\circ}$ explanation for the increased mobility of $\beta 9$ in the absence of RA is that this segment may actually be significantly 
disordered in the partially unstructured intermediate state of CRABP I that serves as a binding template for RA. In this case, $\beta 8$ and $\beta 10$ would actually be in direct contact with each other, being connected by a reverse loop whose residues form the $\beta 9$ strand in the native structure of CRABP I. An important advantage of this conjured structure would be a significant increase of the electrostatic gradient (due to placing the two key basic residues, $\operatorname{Arg}^{111}$ and $\operatorname{Arg}^{131}$, closer to each other) that would guide the RA anion to the ligand-binding site more effectively.

\section{Modulation of Protein Conformational Flexibility by the Ligand}

The presence of RA in solution decreases flexibility of each ${ }^{\circ}$ segment ${ }^{\circ}{ }^{\circ}{ }^{\circ}{ }^{\circ} \mathrm{CRABP}{ }^{\circ},{ }^{\circ}$ as ${ }^{\circ}$ suggested ${ }^{\circ}$ by $^{\circ}$ the ${ }^{\circ}$ reduced HDX $^{\circ}$ rates $^{\circ}\left(\right.$ Figure $\left.^{\circ} 6 \mathrm{~b}\right),{ }^{\circ}$ although ${ }^{\circ}$ the ${ }^{\circ}$ degree ${ }^{\circ}$ of ${ }^{\circ}$ ligandinduced stabilization varies greatly among the protein segments $^{\circ}$ (Figure ${ }^{\circ}$ 7). ${ }^{\circ}$ The $^{\circ}$ most $^{\circ}$ remarkable $^{\circ}$ effect $^{\circ}$ is exerted upon several strands forming one of the two $\beta$-sheets surrounding the solvent-filled cavity of the protein (segments $\beta 3 / 4, \beta 4 / 5, \beta 6$, and $\beta 7$ ). Two $\beta$-turns in this region (3/4 and 5/6) were proposed to be a part of the portal region of CRABP I, a notion that is well supported by the dramatic increase of their stability observed in this work. These two turns contain half of the residues whose side chains make hydrophobic contacts between the ligand and the protein in its native conformation. Most of the other hydrophobic residues that interact with RA in the native conformation of the protein reside within $\alpha \mathbf{I I}$, which is one of the most flexible segments in the apo-protein and exhibits a modest gain in stability in the presence of the ligand.

RA also induces a modest increase of conformational stability of $\beta 10$, a segment that contains two residues forming a salt bridge to the carboxylate group of RA in the holo-form of the protein. Surprisingly, protection of $\beta 8$ increases very insignificantly in the presence of RA, despite the importance of this segment for ligand binding (vide supra). It appears that once the ligand has been guided to the binding site and is further stabilized by multiple hydrophobic contacts, $\operatorname{Arg}^{111}$ becomes somewhat less important for RA retention (electrostatic interaction is primarily mediated by $\mathrm{Arg}^{131}$, whose guanidinium group is located much closer to the carboxylate group of RA in the crystal structure of the holo-protein).

\section{Transient Structural Disorder as an Important Facilitator of Ligand Binding}

The dynamic nature of proteins under native conditions, as well as the functional importance of protein dynamics, is now a well-recognized fact. Perhaps the most illustrious examples of the intimate link between protein dynamics and function are found among the so-called intrinsically unstructured proteins that are

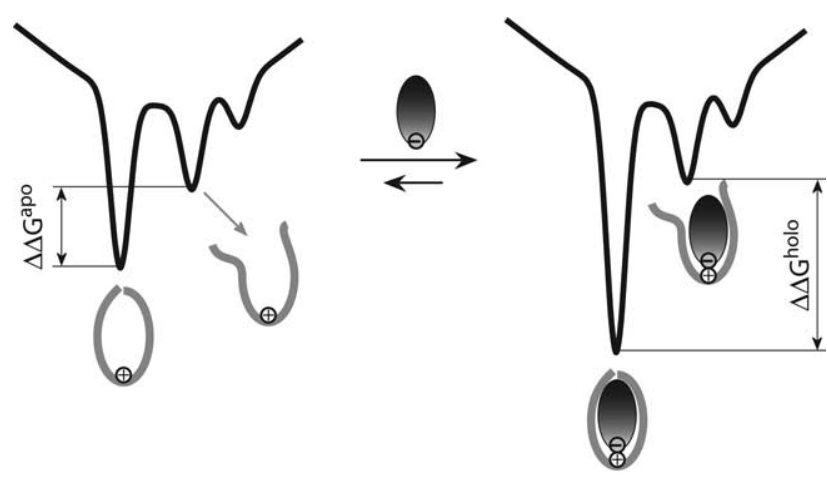

Figure 8. Hypothetical free energy landscapes of CRABP I in the absence (left) and in the presence (right) of RA.

either partially or fully unstructured under native conditions ${ }^{\circ}[29] .{ }^{\circ}$ Proteins $^{\circ}$ that ${ }^{\circ}$ do $^{\circ}$ have ${ }^{\circ}$ native ${ }^{\circ}$ folds ${ }^{\circ}$ under physiological conditions also exhibit dynamic behavior either via local structural fluctuations or by sampling alternative (higher-energy or activated) conformations transiently. Such non-native states are often functionally ${ }^{\circ}$ important ${ }^{\circ}$ despite $^{\circ}$ their ${ }^{\circ}{ }^{\circ}{ }^{\circ}{ }^{\circ} B^{3}$ oltzmann ${ }^{\circ}$ weight $^{\circ}[30$, 31].

The results of the experimental work presented here suggest that such transient disorder is an important facilitator of RA binding to CRABP I. Our observations are consistent with the notion of the apo-form of CRABP I frequently sampling a relatively low-energy partially ${ }^{\circ}$ unstructured ${ }^{\circ}$ equilibrium ${ }^{\circ}$ state $^{\circ}\left(\right.$ Figure $\left.^{\circ} 8\right)$. $^{\circ}$ The core of this intermediate state is formed by strands $\beta 8$ and $\beta 10$, connected by a loop whose residues form strand $\beta 9$ in the native conformation of CRABP I. Additional stabilization of this core region is likely to be provided by strands $\beta 7$ and $\beta 6$ (flanking $\beta 8$ ) and $\beta 1$ (flanking $\beta 10$ ). The stable core generates a significant electrostatic gradient by placing two arginine residues $\left(\mathrm{Arg}^{111}\right.$ and $\mathrm{Arg}^{131}$ ) in close proximity. This electrostatic gradient guides the RA anion to the binding side. While the electrostatic interaction alone can make the proteinligand complex only marginally stable, a transition back to the ground state of the protein traps the ligand within the protein cavity. Furthermore, it brings several hydrophobic side chains in contact with the aliphatic skeleton of RA, thus providing further stabilization of the native conformation. As a result, the free energy of the state corresponding to the global energy minimum becomes even more negative and the sampling of the partially unstructured intermediate state (required for ligand dissociation from the protein) becomes significantly less frequent, effectively trapping RA inside the protein cavity.

The importance of hydrophobic interactions for lowering CRABP I backbone mobility, and as a result, effective ligand retention inside the protein cavity, was also highlighted by the results of our previous work with 9 -cis and 13 -cis isomers ${ }^{\circ}$ of ${ }^{\circ} A^{9}[6]^{\circ}{ }^{\circ}$ Alteration ${ }^{\circ}$ of the aliphatic ${ }^{\circ}$ skeleton ${ }^{\circ}$ of $^{\circ} \mathrm{RA}^{\circ}$ eliminates ${ }^{\circ}$ several ${ }^{\circ}$ "native" hydrophobic contacts, resulting in notable increase of 
the protein HDX rate (compared to exchange carried out in the presence of the cognate ligand, RA). Nevertheless, both 9-cis and 13-cis isomers still bind to CRABP $\mathrm{I}$ and reduce its backbone dynamics (compared to the apo-form of the protein), although not as dramatically

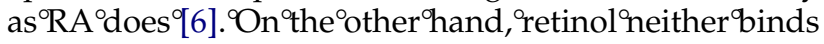
to CRABP I nor induces any change of its backbone dynamics (HDX kinetics of CRABP I is not altered when retinol is present in solution, data not shown). Retinol possesses an aliphatic backbone identical to that of RA, however, it lacks a negatively charged carboxylic group. Therefore, it is incompetent to associate with the transient partially unstructured state of CRABP I via electrostatic interactions, a prerequisite for the proteinligand complex formation.

While the model of RA-CRABP I complex formation proceeding through a partially unstructured state of the protein is well supported by the HDX MS measurements presented in this work, they do not allow the postulated intermediate state to be distinctly detected. Indeed, hydrogen exchange under native conditions occurs $^{\circ}$ in ${ }^{\circ}$ the ${ }^{\circ}$ so-called ${ }^{\circ} \mathrm{EX} 2^{\circ}{ }^{\circ}$ regime ${ }^{\circ}[32]^{\circ}$ and ${ }^{\circ}$ is ${ }^{\circ}$ therefore uncorrelated. ${ }^{\circ}$ Detection ${ }^{\circ}$ and $^{\circ}$ characterization ${ }^{\circ}$ of ${ }^{\circ}$ distinct protein states with HDX MS becomes possible only under ${ }^{\circ} \mathrm{EX}^{\circ}{ }^{\circ}$ conditions $^{\circ}\left(\right.$ e.g., ${ }^{\circ}$ at $^{\circ}$ high $\left.^{\circ} \mathrm{pH}\right),{ }^{\circ}$ which ${ }^{\circ}$ often give $^{\circ}$ rise $^{\circ}$ to $^{\circ}$ correlated $^{\circ}$ exchange $^{\circ}$ pattern ${ }^{\circ}\left[33,{ }^{\circ} 34\right] .{ }^{\circ}$ Preliminary results of HDX MS measurements of CRABP I carried out under conditions favoring EX1 exchange mechanism (pH 10) suggest that the strands $\beta \mathbf{8}$ and $\beta \mathbf{1 0}$ (but not $\beta 9$ ) remain stable in a partially unstructured intermediate state of the protein that is frequently sampled under these conditions (Xiao, unpublished data).

\section{Conclusions}

We have used HDX MS measurements in combination with fragmentation of protein ions in the gas phase to produce backbone stability maps of CRABP I in the absence and in the presence of its cognate ligand, RA. A region of the protein that exhibits significantly higher than average stability in the absence of the ligand contains amino acid residues whose side chains interact with the ligand in the holo-form of the protein to form a salt-bridge, a finding consistent with the results of earlier NMR studies. These protein segments are proposed to form a core of a relatively low-energy, partially unstructured state that is sampled relatively frequently by the apo-protein under native conditions. A significant fraction of the protein sequence in this state is disordered, thus allowing the ligand to have easy access to the binding site, which is restricted in the native (fully structured) state of the protein. The presence of RA in the exchange buffer decreases flexibility of almost all segments of the protein, however, such stabilization is not distributed evenly along the polypeptide chain. The most significant decrease of flexibility is observed within the region that contains a large number of residues whose side chains form hydrophobic contacts with RA in the holo-form of the protein. This region is postulated to be unstructured in the intermediate state of the protein. Multiple hydrophobic contacts between the ligand and the protein stabilize the native conformation, effectively inhibiting transitions to the partially unstructured state (required for RA to leave the confinements of the ligand-binding site). As a result, the ligand becomes effectively trapped by the protein. The proposed model illustrates the functional importance of activated (partially unstructured) protein states despite their low Boltzmann weight (compared with native states). Such transient structural disorder allows the ligand to enter the binding site that is inaccessible in a fully folded state of the protein. Once bound to its captor, the ligand locks it in the native conformation by forming multiple hydrophobic contacts, thus preventing the unlocking of the protein conformation and, consequently, its own release. This work provides strong evidence that RA binding to CRABP I at the endogenous concentration level of the protein does not require dimerization as a means of enhancing ligand access to its binding site inside the internal cavity of CRABP I. This work also illustrates the power of mass spectrometry, which is capable of probing protein dynamics at relatively low concentrations (at or even below the endogenous levels of the protein in tissues) that exclude the possibility of protein self-association and are often inaccessible to other biophysical techniques.

\section{Acknowledgments}

The authors thank Dr. Stephen J. Eyles for helpful discussions, Professor Lila M. Gierasch for providing CRABP I samples, and Dr. Rinat R. Abzalimov for carrying out control experiments on CRABP I aggregation. This work was supported by a grant from the National Institutes of Health (R01 GM61666).

\section{Supplementary Material}

Supplementary data associated with this article can be found, in the online version, at dio:10.1016/ j.jasms.2005.02.020.

\section{References}

1. Banaszak, L.; Winter, N.; Xu, Z.; Bernlohr, D. A.; Cowan, S.; Jones, T. A. Lipid-binding proteins: A family of fatty acid and retinoid transport proteins. Adv. Protein Chem. 1994, 45, 89151.

2. Balmer, J. E.; Blomhoff, R. Gene expression regulation by retinoic acid. J. Lipid Res. 2002, 43, 1773-1808.

3. Dong, D.; Ruuska, S. E.; Levinthal, D. J.; Noy, N. Distinct roles for cellular retinoic acid-binding proteins I and II in regulating signaling by retinoic acid. J. Biol. Chem. 1999, 274, 23695-23698.

4. Donovan, M.; Olofsson, B.; Gustafson, A. L.; Dencker, L.; Eriksson, U. The cellular retinoic acid binding proteins. J. Steroid Biochem. Mol. Biol. 1995, 53, 459-465.

5. Kleywegt, G. J.; Bergfors, T.; Senn, H.; Le Motte, P.; Gsell, B.; Shudo, K.; Jones, T. A. Crystal structures of cellular retinoic acid binding proteins I and II in complex with all-trans- 
retinoic acid and a synthetic retinoid. Structure 1994, 2, 1241-1258.

6. Xiao, H.; Kaltashov, I. A.; Eyles, S. J. Indirect assessment of small hydrophobic ligand binding to a model protein using a combination of ESI MS and HDX/ESI MS. J. Am. Soc. Mass Spectrom. 2003, 14, 506-515.

7. Scapin, G.; Gordon, J. I.; Sacchettini, J. C. Refinement of the structure of recombinant rat intestinal fatty acid-binding apoprotein at 1.2-A resolution. J. Biol. Chem. 1992, 267, 4253-4269.

8. Sacchettini, J. C.; Scapin, G.; Gopaul, D.; Gordon, J. I. Refinement of the structure of Escherichia coli-derived rat intestinal fatty acid binding protein with bound oleate to 1.75-A resolution. Correlation with the structures of the apoprotein and the protein with bound palmitate. J. Biol. Chem. 1992, 267, 2353423545.

9. Jamison, R. S.; Newcomer, M. E.; Ong, D. E. Cellular retinoidbinding proteins: Limited proteolysis reveals a conformational change upon ligand binding. Biochemistry 1994, 33, 2873-2879.

10. Thompson, J. R.; Bratt, J. M.; Banaszak, L. J. Crystal structure of cellular retinoic acid binding protein I shows increased access to the binding cavity due to formation of an intermolecular $\beta$-sheet. J. Mol. Biol. 1995, 252, 433-446.

11. Wang, L.; Li, Y.; Abildgaard, F.; Markley, J. L.; Yan, H. NMR solution structure of type II human cellular retinoic acid binding protein: Implications for ligand binding. Biochemistry 1998, 37, 12727-12736.

12. Wang, L.; Yan, H.NMR study of the binding of all-transretinoic acid to type II human cellular retinoic acid binding protein.Biochim. Biophys. Acta14332401999252

13. Chen, X.; Tordova, M.; Gilliland, G. L.; Wang, L.; Li, Y.; Yan, H.; Ji, X. Crystal structure of apo-cellular retinoic acid-binding protein type II (R111M) suggests a mechanism of ligand entry. J. Mol. Biol. 1998, 278, 641-653.

14. Krishnan, V. V.; Sukumar, M.; Gierasch, L. M.; Cosman, M. Dynamics of cellular retinoic acid binding protein I on multiple time scales with implications for ligand binding. Biochemistry 2000, 39, 9119-9129.

15. Sacchettini, J. C.; Gordon, J. I.; Banaszak, L. J. Crystal structure of rat intestinal fatty-acid-binding protein. Refinement and analysis of the Escherichia coli-derived protein with bound palmitate. J. Mol. Biol. 1989, 208, 327-339.

16. Winter, N. S.; Bratt, J. M.; Banaszak, L. J. Crystal structures of holo- and apo-cellular retinol-binding protein II. J. Mol. Biol. 1993, 230, 1247-1259.

17. Hodsdon, M. E.; Cistola, D. P. Ligand binding alters the backbone mobility of intestinal fatty acid-binding protein as monitored by ${ }^{15} \mathrm{~N}$ NMR relaxation and ${ }^{1} \mathrm{H}$ exchange. Biochemistry 1997, 36, 2278-2290.

18. Hodsdon, M. E.; Cistola, D. P. Discrete backbone disorder in the nuclear magnetic resonance structure of apo intestinal fatty acid-binding protein: Implications for the mechanism of ligand entry. Biochemistry 1997, 36, 1450-1460.
19. Kaltashov, I. A.; Eyles, S. J. Studies of biomolecular conformations and conformational dynamics by mass spectrometry. Mass Spectrom. Rev. 2002, 21, 37-71.

20. Eyles, S. J.; Speir, P.; Kruppa, G.; Gierasch, L. M.; Kaltashov, I. A. Protein conformational stability probed by Fourier transform ion cyclotron resonance mass spectrometry. J. Am. Chem. Soc. 2000, 122, 495-500.

21. Kaltashov, I. A.; Eyles, S. J. Crossing the phase boundary to study protein dynamics and function: Combination of amide hydrogen exchange in solution and ion fragmentation in the gas phase. J. Mass Spectrom. 2002, 37, 557-565.

22. Kaltashov, I. A.; Eyles, S. J.; Xiao, H.2004; Robinson, J. W., Ed.; In Focus on Protein Research; pp 191-218.Nova Science Publishers, Inc.: Hauppauge, NY,

23. Zhang, J.; Liu, Z. P.; Jones, T. A.; Gierasch, L. M.; Sambrook, J. F. Mutating the charged residues in the binding pocket of cellular retinoic acid-binding protein simultaneously reduces its binding affinity to retinoic acid and increases its thermostability. Proteins 1992, 13, 87-99.

24. Burns, L. L.; Dalessio, P. M.; Ropson, I. J. Folding mechanism of three structurally similar $\beta$-sheet proteins. Proteins 1998, 33, $107-118$.

25. Jurchen, J. C.; Garcia, D. E.; Williams, E. R. Gas-phase dissociation pathways of multiply charged peptide clusters. J. Am. Soc. Mass Spectrom. 2003, 14, 1373-1386.

26. Maity, H.; Lim, W. K.; Rumbley, J. N.; Englander, S. W. Protein hydrogen exchange mechanism: Local fluctuations. Protein Sci. 2003, 12, 153-160.

27. Hoerner, J. K.; Xiao, H.; Dobo, A.; Kaltashov, I. A. Is there hydrogen scrambling in the gas phase? Energetic and structural determinants of proton mobility within protein ions. J. Am. Chem. Soc. 2004, 126, 7709-7717.

28. Kim, M. Y.; Maier, C. S.; Reed, D. J.; Deinzer, M. L. Site-specific amide hydrogen/deuterium exchange in E. coli thioredoxins measured by electrospray ionization mass spectrometry. J. Am. Chem. Soc. 2001, 123, 9860-9866.

29. Dunker, A. K.; Brown, C. J.; Lawson, J. D.; Iakoucheva, L. M.; Obradovic, Z. Intrinsic disorder and protein function. Biochemistry 2002, 41, 6573-6582.

30. Tsai, C. D.; Ma, B.; Kumar, S.; Wolfson, H.; Nussinov, R. Protein folding: Binding of conformationally fluctuating building blocks via population selection. Crit. Rev. Biochem. Mol. Biol. 2001, 36, 399-433.

31. Papoian, G. A.; Wolynes, P. G. The physics and bioinformatics of binding and folding-an energy landscape perspective. Biopolymers 2003, 68, 333-349.

32. Krishna, M. M. G.; Hoang, L.; Lin, Y.; Englander, S. W. Hydrogen exchange methods to study protein folding. Methods 2004, 34, 51-64.

33. Hoofnagle, A. N.; Resing, K. A.; Ahn, N. G. Protein analysis by hydrogen exchange mass spectrometry. Annu. Rev. Biophys. Biomol. Struct. 2003, 32, 1-25.

34. Ferraro, D. M.; Robertson, A. D. EX1 hydrogen exchange and protein folding. Biochemistry 2004, 43, 587-594. 